\title{
Biomasa: métodos de producción, potencial energético y medio ambiente
}

Biomass: production methods, energy potential and environment

JULIÁN RODRIGO QUINTERO GONZÁLEZ

Ingeniero en Transporte y Vías Magíster en Ingeniería Ambiental

Docente Facultad de Ingeniería

Universidad Pedagógica y Tecnológica de Colombia jrquinterog.itv@gmail.com, julian.quintero@uptc.edu.co

\section{LAURA ESTEFANÍA QUINTERO GONZÁLEZ}

Ingeniera Ambiental (c)

Universidad Pedagógica y Tecnológica de Colombia

lauraestefania.quintero@uptc.edu.co

Recibido: 19/06/2014

Aceptado: 29/01/2015 


\title{
RESUMEN
}

En este artículo se exponen los conceptos básicos sobre la producción de energía a partir de biomasa, propuestos por varios autores. Se da una visión amplia acerca del papel que desempeña la biomasa en el mercado internacional, mostrando costos, porcentajes y cifras en general, de estudios realizados alrededor del mundo. Se presentan diferentes conceptos y discernimientos sobre el enfoque medioambiental de la biomasa, que varios autores han hecho para evaluar los méritos y/o posibles desventajas de esta tecnología de obtención energética. Finalmente se concluye que existe un interés marcado en todo el mundo por el desarrollo de sistemas de producción de energía a partir de biomasa, a la vez que se tiene especial cuidado en la evaluación de los efectos en el medio ambiente derivados del desarrollo de este tipo de energía y sus tecnologías.

Palabras clave: Biomasa, Potencial Energético, Medio Ambiente.

\begin{abstract}
This document sets out the basic concepts on the production of energy from biomass, proposed by several authors. In this paper, a broad view of the role played by biomass in the international market, showing costs, percentages in general from studies around the world, is given. Different concepts and insights about the environmental approach of the biomass, which several authors have done to assess the merits or possible disadvantages of this technology to obtain energy, are presented. Finally it is concluded that there is a strong interest worldwide in the development of systems of energy production from biomass while special care is taken in the evaluation of the effects on the environment arising from the development of this type of energy and technologies.
\end{abstract}

Keywords: Biomass, Potential Energy, Environment.

Quintero, J. \& Quintero, L. (2015). Biomasa: Métodos de producción, potencial energético y 


\section{INTRODUCCIÓN}

El estudio de potencial energético de la biomasa desde el punto de vista de su aprovechamiento en la producción de combustibles, que eventualmente pudiesen reemplazar a los combustibles fósiles, se ha extendido por todo el mundo, lo cual ha permitido avanzar en el mejoramiento de los procesos de producción a través del perfeccionamiento de las técnicas actuales para el procesamiento de la biomasa. Las investigaciones que ahora son cada vez más comunes, se han enfocado en temas que van desde el estudio de las propiedades químicas y termofísicas de diferentes plantas, pasando por el desarrollo de mejores técnicas de producción hasta la evaluación del potencial energético de lo que podrían llamarse prototipos de combustibles o compuestos de biomasa. Así, el presente artículo tiene por objeto exponer los aspectos más relevantes relacionados con los procesos de producción de biomasa, su potencial energético y los impactos en el medio ambiente derivados de su producción y utilización.

\section{LA BIOMASA}

De acuerdo con Drapcho et ál. (2008), Rudolf Diesel patentó el primer motor diesel en 1892 en Berlín, Alemania, trabajando para Empresas Linde después de mudarse de París. En 1894, presentó el funcionamiento de un motor a escala de casi 3 metros de altura, el cual estalló y por poco acaba con su vida. En 1900, presentó un nuevo motor diesel que trabajaba mediante el uso de aceite de maní como combustible en la Exposición Universal de París. Antes de su misteriosa muerte en 1913, Rudolf Diesel afirmó que: "El uso de aceites vegetales como combustibles para motores puede parecer insignificante hoy, pero estos aceites puede llegar a ser, en el curso del tiempo, tan importantes como el petróleo y los productos de alquitrán de carbón de la actualidad".

\section{Definición de biomasa}

La Unión Europea indica que la "Biomasa es la fracción biodegradable de productos, deshechos y residuos de la agricultura (incluyendo sustancias vegetales y animales), silvicultura e industrias relacionadas, así como la fracción biodegradable de los residuos municipales e industriales” (Cerdá, 2012).

\section{Tipos de biomasa}

De acuerdo con Cerdá (2012) la biomasa puede clasificarse en cuatro tipos así: 


\section{- Biomasa Sólida}

Tiene un aprovechamiento térmico y eléctrico de materia orgánica de origen animal y vegetal, como los cultivos energéticos -aquellos en los que las especies cultivadas tienen como uso específico la producción de energía-, residuos generados en labores de poda de viñedos y frutales generalmente, y residuos de cultivos en invierno.

- Biogás

Obtenido mediante un proceso de fermentación anaeróbica de la materia orgánica producida por bacterias en ambientes faltos de oxígeno. Dicha desgasificación de los residuos puede ser de manera natural en vertederos o inducida en biodigestores. A nivel mundial los líderes en la producción de biogás son Alemania y Reino Unido, teniendo el $61,2 \%$ y 16,2\%, respectivamente, de la producción de la UE, la cual es de 10,9 Mteq (Millones de toneladas equivalentes de petróleo) en 2010.

\section{- Fracción Orgánica de los Residuos Sólidos Urbanos (FORSU).}

Para este tipo de residuos se hace énfasis en la gestión en el proceso de producción de energía que se da en cuatro etapas: 1 . Prevención o reducción de producción de residuos; 2 . Recuperación de residuos por medio del reciclaje, la reutilización y la recolección o cualquier otro proceso que permita extraer materias primas secundarias; 3 . Uso de residuos como fuente de energía; 4 . Vertido de residuos. La utilización de residuos sólidos en la producción de energía evita la generación de gases de efecto invernadero mediante la sustitución de combustibles fósiles y obviando gran parte de emisiones de metano en vertederos.

\section{- Biocarburantes}

Combustibles líquidos cuyas propiedades les permiten sustituir la gasolina en su totalidad o por lo menos en un porcentaje considerable. En la Figura 1 se presenta una síntesis de los diferentes tipos de biomasa y su modo de producción. 


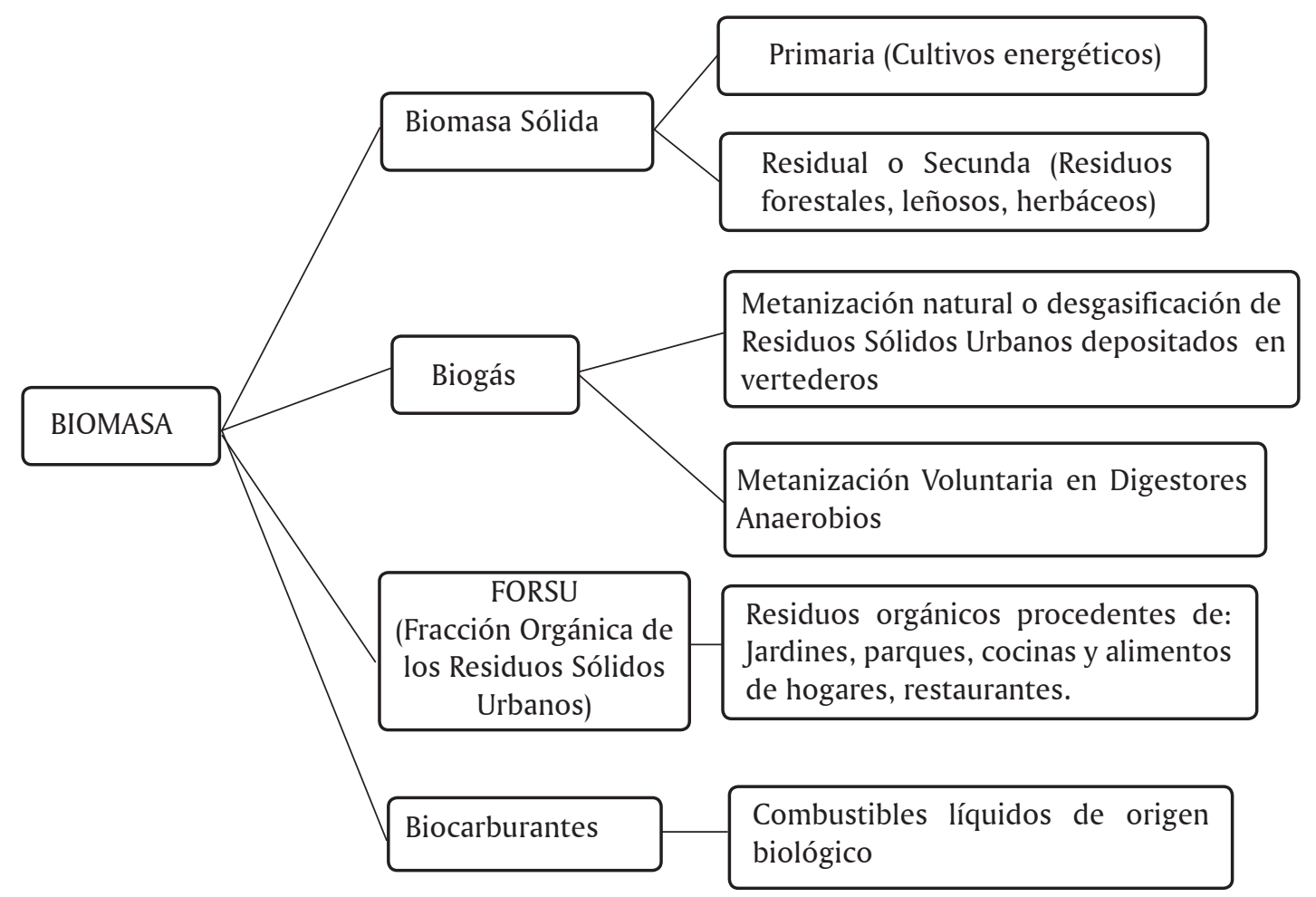

Figura 1. Tipos de biomasa

Fuente: Los Autores.

En cuanto a los usos de la energía obtenida a partir de biomasa, se tienen aplicaciones térmicas como por ejemplo la calefacción o refrigeración de unidades familiares, aunque a nivel industrial existen calderas de biomasa que contribuyen a la producción de calor que en ocasiones se acompaña con la producción eléctrica. El EurObserv'ER registró que en 2010, en la UE la producción de energía primaria a partir de biomasa llegó a 80,1 Mtep teniendo un 75\% aplicaciones térmicas (Cerdá, 2012). A pesar de que la producción eléctrica se puede dar conjuntamente con la producción térmica, el primer tipo precisa sistemas más elaborados en el aprovechamiento de la biomasa, ya que esta suele tener un alto contenido de humedad y de volátiles; sin embargo la gran ventaja de la aplicación eléctrica de la biomasa, en su total disponibilidad y apoyo a la red principal a cualquier hora del día, ya que su producción y suministro no dependen directamente de aspectos climatológicos (Ver Figura 2.). 


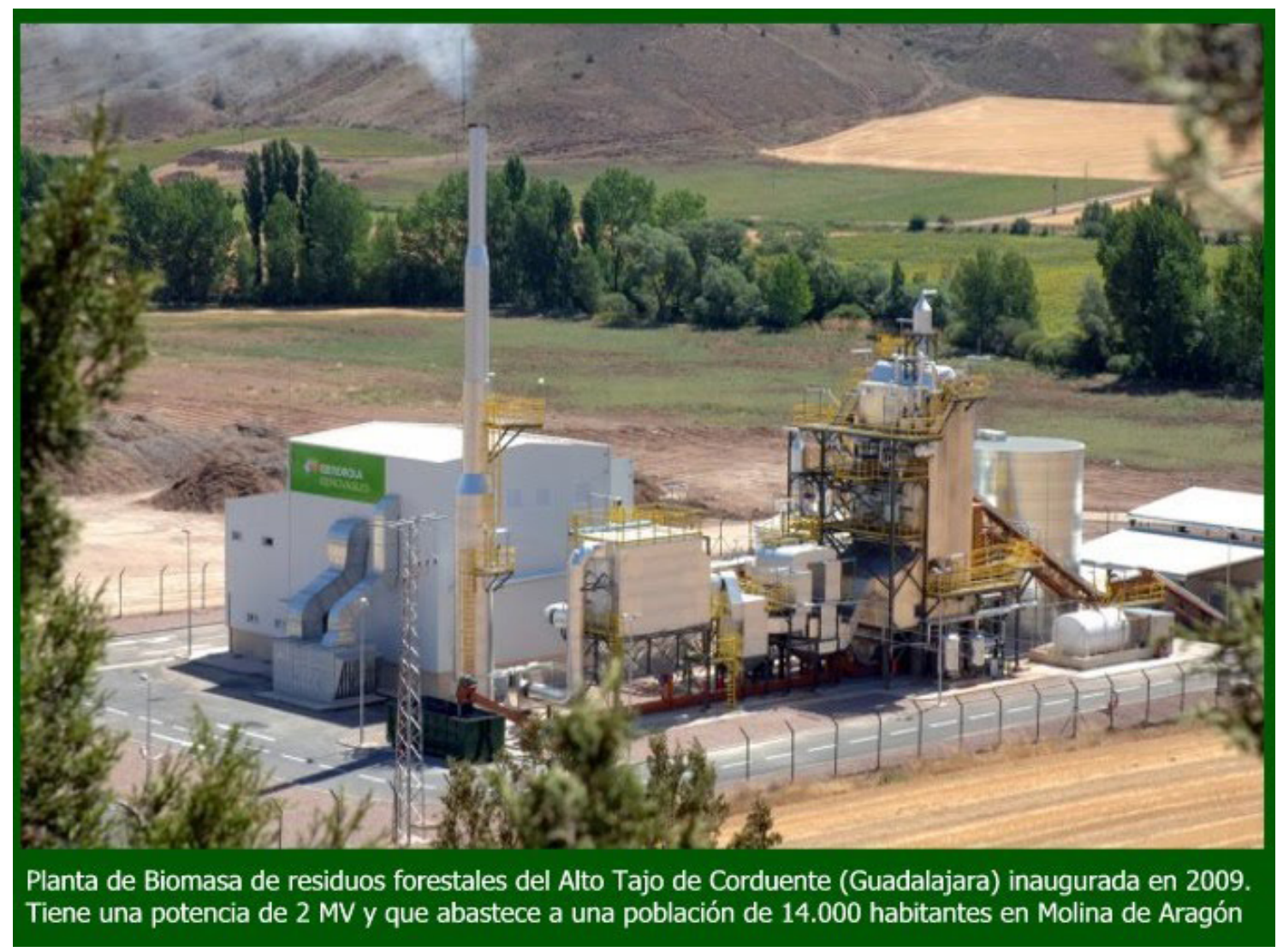

Figura 2. Planta de biomasa de IBERDROLA con producción eléctrica a partir de residuos forestales.

Fuente: http://goo.gl/zcm7NO

\section{MÉTODOS DE PRODUCCIÓN}

De acuerdo con Karaj et ál. (2010) hay tres tipos de fuentes de energía de biomasa, se incluyen los cultivos bioenergéticos, residuos agrícolas y residuos forestales. En la producción energética a partir de biomasa se debe hacer una clara distinción entre el proceso termoquímico que incluye combustión, pirólisis y gasificación, y el proceso bioquímico que reconoce la fermentación y digestión anaerobia para el caso específico de la biomasa (Tabla 1). 


\begin{tabular}{lll}
\hline \hline PROCESO DE CONVERSIÓN & SOLUCIONES TECNOLÓGICAS & \multicolumn{1}{c}{ PRODUCTO FINAL } \\
\hline \hline & Combustión & Vapor \\
& & Procesos de calor \\
& & Energía eléctrica \\
Procesos termoquímicos & Gasificación & Vapor \\
& & Procesos de calor \\
& & Energía eléctrica \\
& & Gas combustible metano \\
& Pirolisis & Carbón \\
& & Bio-carbón \\
Procesos bioquímicos & Fermentación & Gas combustible \\
& & Etanol \\
\hline \hline
\end{tabular}

Tabla 1. Termoquímica y bioquímica de los procesos de clasificación. Fuente: Economics of biomass energy utilization in combustion and gasification plants: effects of logistic variables, Caputo et ál. (2005).

En la combustión de la biomasa intervienen el carbono y el hidrógeno presentes en el combustible, que reaccionan con el exceso de oxígeno dando como resultado $\mathrm{CO}_{2}$, agua y liberación de calor; aunque también existe la cocombustión (combustión de biomasa y carbón) en plantas de generación eléctrica de carbón. Por otro lado, la pirólisis descompone la biomasa mediante una oxidación incompleta en ausencia de oxígeno, generando productos sólidos significativos como carbón vegetal, además de compuestos líquidos y gaseosos para su utilización como combustibles (Figura 3). De igual manera, la gasificación lleva a cabo una oxidación parcial de la biomasa a través del calor, generando finalmente combustibles gaseosos ricos en hidrógeno y $\mathrm{CO}_{2}$. 

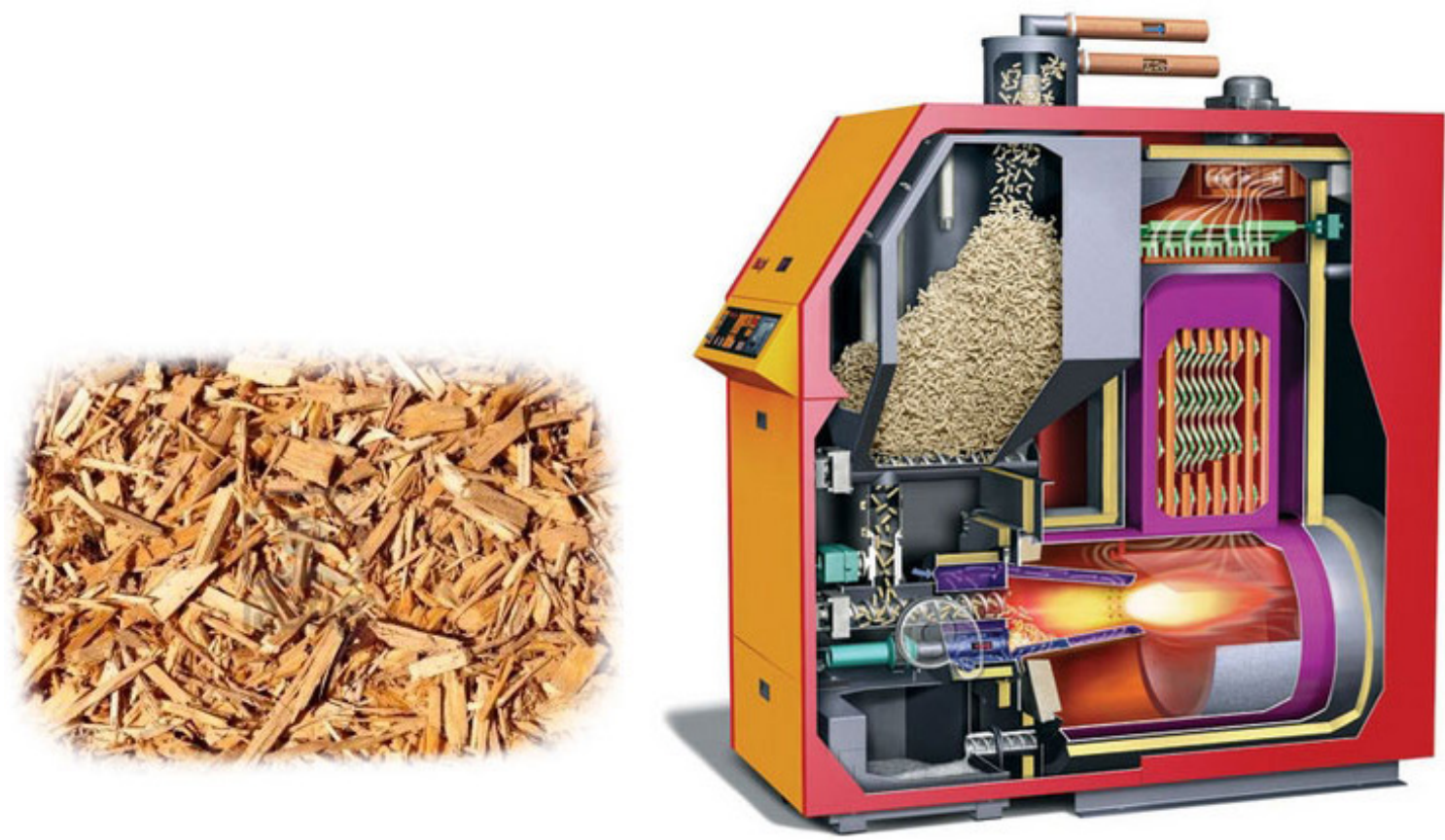

Figura 3. Proceso Termoquímico.

Fuente: http://goo.gl/gs0690

Dentro de los procesos de obtención de energía a partir de la biomasa se encuentra uno del tipo bioquímico que es la digestión anaerobia, en la que la materia orgánica (Estiércol, residuos agrícolas, lodos de aguas residuales, residuos urbanos sólidos o líquidos, residuos de la industria alimentaria) se descompone en biogás mediante grupos de microorganismos en ausencia de oxígeno (Figura 4). 


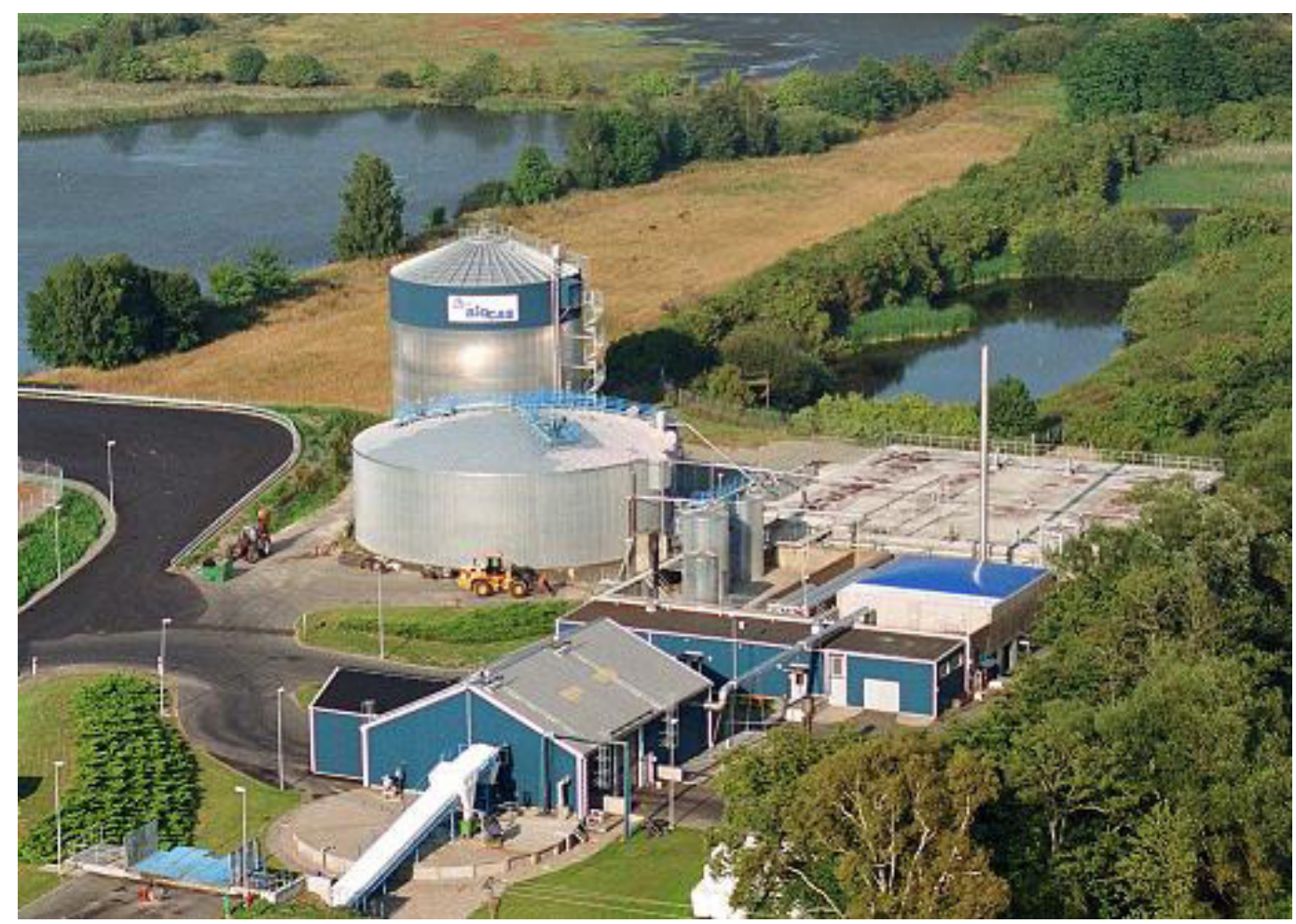

Figura 4. Digestores Anaeróbicos para la producción de biogás.

Fuente: http://goo.gl/jWVGbi

\section{COMERCIO INTERNACIONAL Y POTENCIAL ENERGÉTICO EN COLOMBIA}

\section{Comercio internacional de la biomasa}

Según Heinimö \& Junginger (2009), el comercio internacional de biocombustibles sólidos y líquidos para 2006 fue de 0,9 EJ, de los cuales 0,6 EJ correspondían a comercio indirecto y 0,3 EJ a comercio de etanol, pellets y aceite de palma con fines directamente energéticos; este comercio directo aumentó 60\% en el período de 2004 a 2006. Sin embargo, Heinimö \& Junginger sugirieron un estudio más detallado acerca de estos dos tipos de comercio (directo e indirecto) debido a que son pocos los datos de destino y uso final de cada tipo de combustible. No obstante, hay cifras que indican que el marcado crecimiento de comercio directo de etanol y biodiesel ocurrió en mayor parte en la UE y EE.UU., dando lugar a la exportación de etanol de Brasil, aceite vegetal (palma y soya) y biodiesel del 
sudeste asiático y de Latino América y la exportación de pellets desde Canadá hacia la UE. En la Tabla 2 Cerdá (2012) presenta cifras comparativas de los años 1990 y 2010, que reflejan la Oferta de Energía Primaria y sus porcentajes de origen.

\begin{tabular}{lllll}
\hline \hline & OCDE & OCDE ASIA- & OCDE & OCDE TOTAL \\
& AMÉRICAS (1) & OCEANÍA (2) & EUROPA (3) & \\
\hline 1990 & & & & \\
\hline \hline O.E.P. (en Mtep) & 2.260 & 643 & 1.620 & 4.523 \\
de la cual renovable... & $148,21(6,6 \%)$ & $25,85(4,0 \%)$ & $93,49(5,8 \%)$ & $267,56(5,9 \%)$ \\
de la cual bioenergía... & $77,57(52,3 \%)$ & $9,74(37,7 \%)$ & $50,09(53,6 \%)$ & $131,41(51,4 \%)$ \\
\%biom. sól./bioen. & $96,3 \%$ & $96,8 \%$ & $93,7 \%$ & $95,4 \%$ \\
\%biocarb./bioen. & -- & -- & -- & -- \\
\%biogás./bioen. & $1 \%$ & $1,1 \%$ & $1,4 \%$ & $1,1 \%$ \\
\%re. mu. re.(4)/bioen. & $2,8 \%$ & $2,1 \%$ & $4,9 \%$ & $3,5 \%$ \\
2010 (estim.) & & & 1.814 & 5.413 \\
O.E.P. (en Mtep) & 2.691 & 907 & $188,88(10,4 \%)$ & $411,94(7,6 \%)$ \\
de la cual renovable... & $191,66(7,1 \%)$ & $31,40(3,52 \%)$ & $235,12(57,1 \%)$ \\
de la cual bioenergía... & $109,31(57,03 \%)$ & $12,45(39,7 \%)$ & $113,36(60,0 \%)$ & $69,4 \%$ \\
\%biom. sól./bioen. & $66,5 \%$ & $81,6 \%$ & $70,8 \%$ & $18,1 \%$ \\
\%biocarb./bioen. & $24,8 \%$ & $4,6 \%$ & $13,2 \%$ & $6,7 \%$ \\
\%biogás./bioen. & $5,1 \%$ & $4,8 \%$ & $8,5 \%$ & $5,8 \%$ \\
\%re. mu. re./bioen. & $3,7 \%$ & $9 \%$ & $7,5 \%$ & \\
\hline \hline
\end{tabular}

(1) OCDE Américas: Canadá, Chile, México y Estados Unidos.

(2) OCDE Asia-Oceanía: Australia, Israel, Japón, Corea y Nueva Zelanda.

(3) OCDE Europa: Austria, Bélgica, República Checa, Dinamarca, Estonia, Finlandia, Francia, Alemania, Grecia, Hungría, Islandia, Italia, Luxemburgo, Holanda, Noruega, Polonia, Portugal, Eslovaquia, Eslovenia, España, Suecia, Suiza, Turquía y Reino Unido.

(4) Residuos Municipales Renovables.

Tabla 2. Oferta de energía primaria total, de energía renovable y de bioenergía en la Organización para la Cooperación y el Desarrollo Económicos OCDE, por regiones del mundo y total en 1990 y 2010.

Fuente: Energía obtenida a partir de biomasa, Cerdá (2012). 
Al hablar de cualquier actividad de producción es evidente que esta generará costos, y la actividad de producción energética a partir de biomasa no es la excepción. En este proceso se generan otras actividades como transporte de materia prima, combustibles, equipos y tecnología, entre otros. En la Tabla 3 se muestra un compilado de datos sobre rendimiento (GJ/ha/año: Giga Julio por Hectárea por año) y costos (US\$ ${ }_{2005} / \mathrm{GJ}$ : Dólar por Giga Julio en el año 2005) de diferentes materias primas en la producción de bioenergía.

\begin{tabular}{|c|c|c|c|}
\hline MATERIA PRIMA & REGIÓN & RENDIMIENTO GJ/HA/AÑO & COSTO US\$2005/GJ \\
\hline \multicolumn{4}{|c|}{ CULTIVOS ENERGÉTICOS } \\
\hline Miscanthus & Europa & $190-280$ & $4,8-16$ \\
\hline $\begin{array}{l}\text { Paricum virgatum } \\
\text { (Switchgrass) }\end{array}$ & Europa & $120-225$ & $2,4-3,2$ \\
\hline $\begin{array}{l}\text { Paricum virgatum } \\
\text { (Switchgrass) }\end{array}$ & Norte de América & $103-150$ & 4,4 \\
\hline Árboles rotación corta & Sur de Europa & $90-225$ & $2,9-4$ \\
\hline Eucaliptus & Sur de Europa & $150-415$ & 2,7 \\
\hline Sauce (rotación corta) & Europa & 140 & 4,4 \\
\hline Astillas madera & Europa & 110 & $3,4-13,6$ \\
\hline Astillas madera & Centro de América & $80-150$ & $1,8-2$ \\
\hline \multicolumn{4}{|c|}{ RESIDUOS PRIMARIOS } \\
\hline Paja de trigo & Europa & 60 & 1,9 \\
\hline Paja de trigo & USA & $7-75$ & N.D. \\
\hline Paja de azúcar de caña & Brasil & $90-126$ & N.D. \\
\hline Cañote de maíz & Norte de América & $15-155$ & N.D. \\
\hline Cañote de maíz & India & $22-30$ & 0,9 \\
\hline Rastrojo de sorgo & Mundo & 85 & N.D. \\
\hline Residuos forestales & Europa & $2-15$ & $1-7,7$ \\
\hline
\end{tabular}

Tabla 3. Rendimientos y costos de producción y recolección de diferentes materias primas de biomasa en el mundo. Fuente: Energía obtenida a partir de biomasa, Cerdá (2012). 


\section{Potencial energético en Colombia}

En relación al potencial energético se destaca el análisis preliminar de biomasa potencialmente útil para obtener diesel, desarrollado por Cabrera et ál. (2011); en la región de la Zona Pacífica de Colombia se realizó una comparación entre las propiedades termo-físicas de los biodiesel y las propiedades del diesel comercial, desarrollando pruebas de rendimiento de estos biocombustibles en los motores de encendido por compresión evaluando la eficiencia, el consumo y la potencia a diferentes revoluciones por minuto (RPM) del motor. Los combustibles probados fueron los siguientes: el Biodiesel A compuesto por Elaeis Oleífera (Palma Americana de Aceite), el Biodiesel B por Elaeis Guineensis (Palma Africana de Aceite), el Biodiesel C por Ricinus Comunis (Ricino o Higuera Infernal) y los Biodiesel 1 y 2 compuestos por aceite de palma y tratados con metanol y etanol respectivamente (Cabrera et ál., 2011).

Los resultados permitieron establecer que los biodiesel presentaban eficiencias cercanas a las del diesel comercial (Tabla 4), además de potencias evaluadas a diferentes revoluciones por minuto del motor (Tabla 5), la potencia del combustible tipo diesel, a mayores revoluciones por minuto, fue casi equiparada por los biodiesel, dejando en evidencia la bondad de este tipo de combustible y su posibilidad de reemplazar a los combustibles convencionales en el corto plazo. Sin embargo, se resalta el poder calorífico inferior y diferencias en la viscosidad frente al diésel (Cabrera et ál., 2011).

\begin{tabular}{lllllll}
\hline \hline $\begin{array}{l}\text { REVOLUCIONES } \\
\text { POR MINUTO RPM }\end{array}$ & BIODIESEL A & BIODIESEL B & BIODIESEL C & BIODIESEL 1 & BIODIESEL 2 & DIESEL COMERCIAL \\
\hline \hline 750 & $9,07 \%$ & $10,50 \%$ & $9,36 \%$ & $10,32 \%$ & $9,97 \%$ & $14,74 \%$ \\
1300 & $6,04 \%$ & $6,93 \%$ & $5,73 \%$ & $6,81 \%$ & $6,10 \%$ & $9,00 \%$ \\
1750 & $5,40 \%$ & $5,72 \%$ & $5,12 \%$ & $5,63 \%$ & $5,45 \%$ & $7,55 \%$ \\
1950 & $4,31 \%$ & $4,40 \%$ & $4,09 \%$ & $4,32 \%$ & $4,35 \%$ & $5,21 \%$ \\
230 & $4,10 \%$ & $4,00 \%$ & $3,97 \%$ & $4,01 \%$ & $4,22 \%$ & $4,55 \%$ \\
\hline \hline
\end{tabular}

Tabla 4. Eficiencias estimadas de diferentes tipos de biodiesel frente a diesel comercial, Zona del Pacífico Colombiano. Fuente: Preliminary analysis of biomass potentially useful for producing biodiesel, Cabrera et ál., 2011. 


\begin{tabular}{ccccccc}
\hline $\begin{array}{c}\text { REVOLUCIONES POR } \\
\text { MINUTO RPM }\end{array}$ & BIODIESEL A & BIODIESEL B & BIODIESEL C & BIODIESEL 1 & BIODIESEL 2 & DIESEL COMERCIAL \\
\hline \hline 750 & 7,09 & 6,92 & 6,75 & 6,81 & 7,49 & 8,52 \\
1300 & 11,97 & 12,42 & 11,39 & 17,71 & 17,71 & 20,66 \\
1750 & 20,15 & 20,01 & 19,16 & 27,32 & 27,62 & 31,79 \\
1950 & 23,44 & 24,20 & 22,29 & 35,42 & 35,42 & 39,35 \\
230 & 38,37 & 39,44 & 36,49 & 47,01 & 44,92 & 50,14 \\
\hline \hline
\end{tabular}

Tabla 5. Potencias en HP estimadas de diferentes tipos de biodisel frente a diesel comercial, Zona del Pacífico Colombiano.

Fuente: Preliminary analysis of biomass potentially useful for producing biodiesel, Cabrera et ál., 2011.

\section{MEDIO AMBIENTE}

De acuerdo con Hall \& Scrase (1998) muchas organizaciones y entidades en todo el mundo prevén que la producción de energía mediante biomasa juega un papel importante en una matriz de energías sostenibles para el futuro. Países como Austria, Brasil, Dinamarca, Finlandia, Suecia, India, Estados Unidos y el Reino Unido están promoviendo el uso activo de biomasa para la energía y el impulso del desarrollo de los conocimientos y la tecnología necesarios para los sistemas de energía de biomasa avanzados. Existe un consenso creciente de que la energía renovable debe desplazar progresivamente el uso de combustibles fósiles, con los temores del cambio climático global añadiendo urgencia a esta necesidad.

Desde muchos puntos de vista, las energías renovables se consideran "amigables con el medio ambiente", frase que se ha acuñado para aquellos mecanismos de producción de energía que generan un bajo agotamiento de los recursos naturales, bajas emisiones de energía y en general bajos impactos a los recursos de la tierra; suelo, agua, aire, flora y fauna. En este aspecto la energía producida a partir de biomasa es renovable, pero comparte muchas características con los combustibles fósiles. Los biocombustibles pueden ser transportados y almacenados, y permiten la generación de calor y energía que es esencial en comparación con la alta dependencia de las fuentes intermitentes como la eólica. Un aspecto en común con los combustibles fósiles son los impactos ambientales incluyendo las emisiones al aire, considerado como el más importante en la etapa de conversión.

La energía fósil es a menudo rechazada por razones ambientales, y por lo tanto, es esencial que se evalúe el perfil ambiental de las tecnologías que se propongan para reemplazarla, para lo cual han surgido diversos métodos que se puede utilizar para evaluar los méritos medioambientales relativos de las diferentes opciones de energía, ejemplo de estos son el análisis del ciclo de vida (LCA; Life-Cycle Analysis) y la estimación de los costos externos. Estos métodos han aportado información valiosa 
que según Hall \& Scrase deben evaluar tres consideraciones importantes a tener en cuenta cuando se trata de evaluar el perfil ambiental de la energía de biomasa.

(a) La energía producida a partir de la biomasa es diversa y sus impactos se generan de acuerdo al sitio específico en que se emplace el proyecto. Las materias primas pueden ser cultivadas o subproductos, puede ser leñosa o herbácea, cultivos de azúcar o estiércol y pueden ser tratadas para producir combustibles sólidos, líquidos o gaseosos. Estos a su vez pueden ser utilizados de diversas maneras para la generación de calor y electricidad o en el transporte. Debido a esta situación un solo indicador compuesto no podría expresar el impacto ambiental de la energía de biomasa en general.

(b) El desarrollo de los sistemas de energía de la biomasa moderna está en una etapa relativamente temprana, gran parte de la I+D (Investigación y Desarrollo) en el campo de la energía a partir de biomasa se centra en el desarrollo de la oferta de combustible y la búsqueda de procesos de conversión que minimicen los impactos ambientales tales como la tecnología de cultivos leñosos de alto rendimiento y la generación de energía eficiente mediante gasificación.

(c) Cualquier intento de llegar a un indicador compuesto de desempeño ambiental, incluso con el propósito de definir diferentes opciones o alternativas implica consideraciones subjetivas sobre los impactos ambientales. El peso relativo dado a la acidificación, el calentamiento global o la erosión del suelo dependerá del criterio de cada analista o ingeniero, la zona de influencia y las preferencias, y puede cambiar con el tiempo. Cuando se habla de los impactos ambientales referenciados de experiencias en otros países, se debe ser cuidadoso en su análisis, pues cada proyecto presentará condiciones diferentes.

Berndes et ál. (2003) hicieron una revisión de 17 artículos sobre el suministro futuro de biomasa, dando como conclusiones cifras muy variadas debido a que los niveles de rendimiento de la tierra son muy inciertos y dependen de muchos otros factores variables, así por ejemplo, la estimación de acceso a biomasa más alta para el año 2050 es 9 veces mayor que la estimación más baja. Esta información tan variada se presenta porque los estudios analizados no arrojaban datos acerca de la relación del uso de la tierra con fines energéticos y otros usos como la industria alimentaria, por tal razón no se pudo concluir claramente sobre las consecuencias socioeconómicas de la actividad bioenergética. Además estos estudios, según Berndes et ál., no visualizan las afectaciones de los potenciales energéticos de la biomasa en la biodiversidad de los hábitats. Por esto, Berndes termina por concluir que es necesaria la evaluación de estos aspectos en presencia del aprovechamiento de la biomasa, para saber qué tanto llegan a armonizar la bioenergía a gran escala, el uso de la tierra y la adquisición de la materia prima.

En el 2011, Abbas et ál. hablan acerca de los aspectos ambientales a tener en cuenta en la recolección de biomasa forestal, donde para los autores la producción y extracción de esta puede impactar el 
suelo, la hidrología y la calidad del agua y los recursos del hábitat; por lo que estos criterios deben ser estudiados y en constante actualización por entidades científicas, debido a que solo existen parámetros en lo que respecta al cultivo y regeneración de especies energéticas mas no de las afectaciones del entorno por la producción de biomasa.

Recientemente en Reino Unido Keirstead et ál. (2012), evaluaron las alternativas bioenergéticas para un eco-ciudad. Los resultados arrojaron que la biomasa promete ser una opción en el abastecimiento de energía con emisiones bajas de carbono para una ciudad. Sin embargo, el abastecimiento de biomasa se haría en mayor parte mediante importaciones, lo que eleva los costos de transporte y procesamiento en el lugar. El impacto ambiental de esta alternativa les fue difícil de determinar puesto que las rutas de transporte de biocombustibles alterarían el ciclo de vida y las ventajas de las tecnologías de conversión de biomasa en el centro urbano se especifican según la geografía de la región, aunque queda claro que estas disminuyan las emisiones de carbono significativamente. Cabe resaltar que el modelo de eco-ciudad planteado por los autores se basa en el coste mínimo de capital, incluyendo costes de redes de transporte de biomasa y tecnologías de conversión y la importación de ciertos combustibles.

Finalmente, en el mismo año, Pedroli B. et ál. (2012) se cuestionan si en Europa está surgiendo energía verdaderamente compatible con la biodiversidad, concluyendo que la producción de biomasa para gran demanda bioenergética provoca una variación constante en los hábitats y producirá una pérdida de la biodiversidad, así por ejemplo, según Pedroli, en Europa central el uso de tierras agrícolas para monocultivos representa un riesgo para la estabilidad territorial, en el norte de Europa al retirar la biomasa existente en la superficie de los bosques (madera muerta) disminuye la población de organismos presentes en el suelo y la actividad microbiana y en países como Bélgica y Dinamarca la utilización de estiércol animal produce la acidificación del medio gracias a la producción de amoniaco $\left(\mathrm{NH}_{3}\right)$.

\section{CONCLUSIONES}

En las dos décadas siguientes a los 90's, el uso de la biomasa como biocombustible ha aumentado en el mundo, superando al uso como biogás. Este aumento en el uso vino acompañado, por supuesto, de estudios de potencial energético, rendimiento y medio ambiente derivados del desarrollo de diferentes tipos de biomasa tales como la biomasa sólida, el biogás, la fracción orgánica de los residuos sólidos urbanos (FORSU) y los biocarburantes. Esto permite evidenciar el interés de los países en el desarrollo de sistemas producción de energía a partir de biomasa y la inclusión de este tipo de energías en sus nuevas políticas ambientales, económicas y de desarrollo sostenible especialmente en Asia, Oceanía, Europa, Norteamérica y Latinoamérica donde actualmente se cuenta con un alto potencial energético. 
Desde la perspectiva medioambiental, la biomasa como alternativa de energía limpia, tiene grandes ventajas al disminuir la huella de carbono, aunque también representa la modificación de hábitats con la recolección de la materia prima, sin embargo teniendo en cuenta los diversos tipos, usos, y procesos de conversión de la biomasa, el estudio del impacto ambiental debe ser personalizado y ajustado a cada caso, no se puede hacer una generalización de esta técnica energética y su intervención en el medio ambiente. Así como las desventajas son inexactas, también lo es el tiempo y cantidad de suministro, puesto que no existen cifras claras del uso de la tierra, alimentación o biomasa.

\section{REFERENCIAS BIBLIOGRÁFICAS}

Abbas, D., Current, D., Phillips, M., Rossman, R., Hoganson, H. \& Brooks, N. (2011). Guidelines for harvesting forest biomass for energy: A synthesis of environmental considerations. Biomass and Bioenergy, International Journal. No. 35, pp. 4538-4546, 9 p.

Berndes, G., Hoogwijk, M. \& Van Den Broek, R. (2003). The contribution of biomass in the future global energy supply: a review of 17 studies. Biomass and Bioenergy, International Journal, No. 25, pp. $1-28,28 \mathrm{p}$.

Cabrera Cifuentes, G., Burbano Jaramillo, J.C. \& García Melo, J.I. (2011). Preliminary analysis of biomass potentially useful for producing biodiesel. Dyna, Journal. Year 78, Nro. 170, pp. 144-151, ISSN 0012-7353. Medellín, Colombia. Diciembre, 2011.

Caputo, A.C., Palumbo, M., Pelagagge, P.M. \& Scacchia, F. (2005). Economics of biomass energy utilization in combustion and gasification plants:effects of logistic variables. Biomass and Bioenergy, International Journal. No. 28, pp. 35-51, 17 p.

Cerdá, E. (2012). Energía obtenida a partir de biomasa. Cuadernos económicos de ICE. Núm. 83, 2012, ISSN 0210-2633, pp. 117-140, 24 p. Madrid, España.

Drapcho, C.M., Phu Nhuan, N. \& Walker, T.H. (2008). Biofuels Engineering Process Technology. McGraw-Hill Companies, Inc. 351 p. United States of America.

Hall, D.O. \& Scrase, J.I. (1998). Will biomass be the environmentally friendly fuel of the future? Biomass and Bioenergy, International Journal. Vol. 15, Nos. 4/5, pp. 357-367, 11 p.

Heinimö, J. \& Junginger, M. (2009). Production and trading of biomass for energy - An overview of the global status. Biomass and Bioenergy, International Journal. Vol. 33, pp. 1310-1320, 11 p.

Karaj, Sh., Rehl, T., Leis, H. \& Muller, J. (2010). Analysis of biomass residues potential for electrical energy generation in Albania. Renewable and Sustainable Energy Reviews, Journal, No. 14, pp. 493-499, 7 p. 
Keirstead, J., Samsatli, N., Pantaleo, A.M. \& Shah, N. (2012). Evaluating biomass energy strategies for a UK eco-town with an MILP optimization model. Biomass and Bioenergy, International Journal, No. 39, pp. 306-316, 11 p.

Pedroli, B., Elbersen, B., Frederiksen, P., Grandin, U., Heikkilä, R., Henning Krogh, P., Izakovicová, Z., Johansen, A., Meiresonne, L. \& Spijker, J. (2012). Is energy cropping in Europe compatible with biodiversity? - Opportunities and threats to biodiversity from land-based production of biomass for bioenergy purposes. Biomass and Bioenergy, International Journal. Volume 55, August 2013, pp. $73-86,14 \mathrm{p}$. 


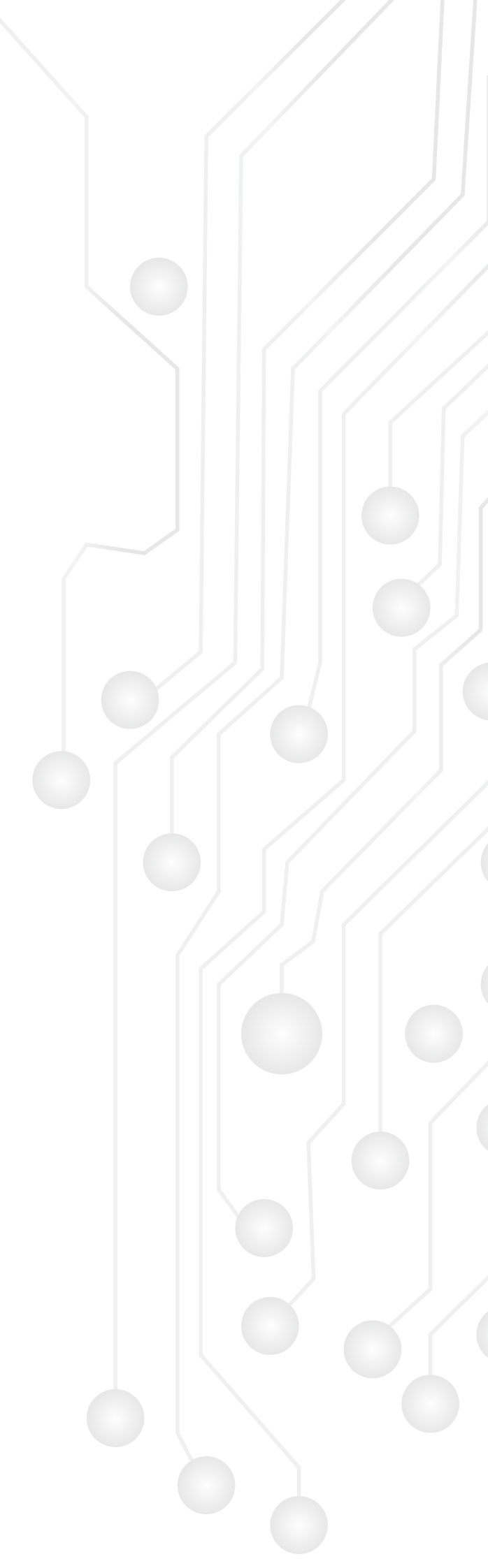

